\section{Las enfermedades periodontales necrotizantes Parte I}

\author{
Necrotizing periodontal diseases. Part. I
}

\begin{abstract}
Resumen:
Las enfermedades periodontales necrotizantes según el Taller Internacional para la Clasificación de la Enfermedades y Condiciones Periodontales realizado en 1999, son dos; la Gingivitis Necrotizante (GN) y la Periodontitis Necrotizante (PN). Una variación más agresiva, que puede estar ligada a las dos entidades mencionadas es la Estomatitis Necrotizante (EN). En esta revisión trataremos sus definiciones, etiología, aspectos clínicos, diagnóstico y tratamiento, tratando de aclarar estos puntos con conceptos actualizados.

Palabras clave: Gingivitis necrotizante, Periodontitis necrotizante, Estomatitis necrotizante, características clínica, diagnóstico y tratamiento.

Abstract:

Necrotizing periodontal diseases according to the International Workshop for Classification of Periodontal Diseases and Conditions in 1999 are two: Necrotizing gingivitis (NG) and Necrotizing periodontitis (NP). Necrotizing stomatitis (NE) is a more aggressive variation, which can be linked to the two entities mentioned. In this review we discuss their definitions, etiology, clinical aspects, diagnosis and treatment, trying to clarify these points with updated concepts.

Keywords: necrotizing gingivitis, necrotizing periodontitis, necrotizing stomatitis, features clinical, diagnosis and treatment.
\end{abstract}

Artículo De Revisión

\author{
Luis Maita Veliz ${ }^{1}$, Donald Ramos \\ Perfecto $^{2}$, Luis M. Maita Castañeda ${ }^{3}$ \\ Luis Hernando Gálvez Calla²
}
1 Departamento Académico Médico Quirúrgico, Facultad de Odontología, Universidad Nacio- nal Mayor de San Marcos. Perú.
2 Departamento Académico de Ciencias Básicas, Facultad de Odontología, Universidad Nacio- nal Mayor de San Marcos. Perú.
3 Facultad de Odontología, Universidad San Martín de Porres. Perú.

\section{Correspondencia:}

Dr. Luis Maita Veliz

Facultad de Odontología, Universidad Nacional Mayor de San Marcos. Av. Germán Amezaga s/n, Lima 1. Perú.

Correo electrónico: luismv21@yahoo.com

Fecha de recepción: 04-12-13

Fecha de aceptación: 19-06-14

\section{Introducción}

Las enfermedades periodontales necrotizantes, tienen precedente en la historia. Se tiene reportes que hace 400 año A.C., los soldados del ejército griego, se quejaban de tener dolor, ulceras y mal olor de sus bocas ${ }^{1}$. Ya en principios del siglo XX, Gilmer manifiesta que las personas con Gingivitis Ulcero necrotizante (GUN), presentaban casi siempre daño de la papila interdental ${ }^{2}$. Lo que actualmente se tiene como consenso, es que estas enfermedades, tiene un patrón general común: dolor intenso, ulceración de tejidos con necrosis, así como un sangrado espontáneo al mínimo estímulo ${ }^{3,4,5,6,7,8}$ Otras manifestaciones no tan esenciales son la halitosis, seudomenbrana, fiebre,

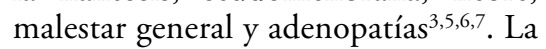
Academia Americana de Periodoncia (AAP) clasifica las enfermedades Periodontales necrotizantes en dos; la Gingivitis necrotizante (GN) y la Periodontitis necrotizante $(\mathrm{PN})$, esta última secuela de la primera ${ }^{3,5,9,10}$. Una variación más agresiva que daña tejido por fuera de los límites mucogingi- vales y a profundidad es la Estomatitis necrotizante (EN), también llamada Noma $^{5,7,11,12}$ que por su mayor invasibilidad tiende a destruir tejidos intra y extraorales ${ }^{11,12}$. Estas enfermedades son insidiosas y se observan factores predisponentes, como el abuso del alcohol y tabaco, desnutrición, inmunosupresión, estrés emocional, gingivitis preexistente y otros cuadros debilitantes $^{4}$. Su prevalencia es baja en países desarrollados $0.5 \%$ y se incrementa hasta $26.9 \%$ a más en los países subdesarrollados como el África ${ }^{13}$, donde se presentan los casos más graves, como el Noma ${ }^{11}$, su aparición por lo general en edades tempranas(niños y jóvenesadultos) siendo asociado a una desnutrición severa ${ }^{6}$.

En relación a su evolución, las enfermedades periodontales necrotizantes, son rápidamente progresivas, destruyendo tejidos desde los gingivales hasta el óseo, teniendo una secuencia de estadios según distribución del área de necrosis; que inician con la necrosis del extremo de la papila y continua con necrosis completa de papila, ne- crosis que afecta la encía marginal, necrosis que afecta la encía adherida, necrosis que llega a la mucosa labial o yugal, exposición del hueso alveolar y perforación de la cara. Estos estadios serian el paso de una gingivitis necrotizante a una estomatitis necrotizante ${ }^{14,15}$.

Gingivitis necrotizante (GN) Sinónimos: "gingivitis ulceromembranosa","gingivoestomatitis de Vincent", "gingivitis ulcero necrotizante aguda", "estomatitis vesiculomembranosa", "infección fusoespiral", "boca de trinchera".

\section{Definición:}

Es un Cuadro inflamatorio, caracterizado por ulceración y necrosis de la papila interdental, el dolor es de moderado a severo, sangrado espontaneo o de fácil provocación al estímulo. La lesión papilar suele ser a sacabocado, pudiendo haber una membrana amarilla grisácea que cubre la lesión, así como halitosis, fiebre y adenopatías a nivel cervical. $3,5,6,7,16,17$ 


\section{Etiología}

Son varios los factores a considerar para su etiología uno de ellos el factor microbiano, importante pero no determinante. Juega un papel fundamental el factor susceptibilidad del huésped, factores predisponente como el tabaco, alcohol, mala higiene bucal, estrés psíquico, desnutrición, deficiencia vitamínica, alteraciones hematológicas y endocrinas, pueden ayudar a su aparición, pero el factor biofilm es uno de los de mayor transcendencia. ${ }^{5}$

\section{Fisiopatología}

La lesión se inicia por la existencia marcada de microorganismos, que tratan de romper la homeostasis en el surco gingival, se han reportado la predominancia de bacterias anaeróbicas gram negativas, que por presentar endotoxinas, pueden provocar activación del complemente por vía alternativa, liberando quimiotoxinas, activando macrófagos y linfocitos $\mathrm{T}$ y $\mathrm{B}$, lo que interfiere con las citoquinas producidas por estas células. Así también estas endotoxinas, estimulan procesos catabólicos al degradar el conectivo y el hueso, producen lesiones directamente en las células y vasos generando necrosis, característica de esta lesión ${ }^{5}$.Se tiene una participación importante del Treponema denticola. Esta espiroqueta gram negativa, coloniza la zona por medio de su proteína $M s p$ y usa sus endoflagelos para dirigirse a profundidad, así como usa su enzima característica la dentilisina, para aumentar la permeabilidad, permitiendo una mejor invasión y su acción proteolítica genera daño a células y sustratos como colágeno, laminina, Ig G. ${ }^{18}$

\section{Microbiología}

La flora microbiana se puede dividir en una flora constante, constituida por Treponema Sp, Fusobacterium sp, Selenomonas sp y Prevotella intermedia y una flora variable, compuesta por una cantidad heterogenia de bacterias como Leptotrichia buccalis y Porphyromonas. ${ }^{7}$

\section{Aspectos clínicos}

Son componentes prodrómicos de la enfermedad, dolor, lesiones a sacabocados con necrosis de la papila interdental y sangrado espontáneo, otras no tan esenciales, halitosis ${ }^{19}$, seudomembrana, fiebre, malestar general, linfoadenopatías $^{5,6,7}$.

\section{Diagnóstico}

El diagnóstico se basa en la información dada por el paciente y de los aspectos clínicos que ya se mencionaron. Para el diagnóstico diferencial se debe evitar confundir la lesión con las siguientes entidades: estomatitis aftosa recurrente, gingivoestomatitis herpética primaria, mucositis microbiana, eritema exudativo multiforme, gingivitis descamativa, gingivitis gonocócica, abscesos periodontales y otros $3,5,6,7,20$.

\section{Tratamiento}

Realizado en 2 tiempos:

1. Tratamiento inmediato, a la fase aguda de la enfermedad, procediendo a una limpieza superficial de la zona con clorhexidina al $0.12 \%$ ó peróxido de hidrogeno al 3\%, no ser invasivos, reducir a su mínima expresión el biofilm supragingival y de forma moderada la subgingival. ${ }^{1,4,5,6}$ Para el dolor un analgésico $\mathrm{o}$ antiinflamatorio como el Ibuprofeno de $400-600 \mathrm{mg}$. c/ 8 horas $^{21}$ por 2-3 días, indicar uso de colutorio a base de clorhexidina al $0.12 \% 2$ veces/día x 10 días, luego usar la de $0.05 \%$. Se indicara antibiótico si el paciente presenta fiebre, malestar general, linfoadenopatias, pudiendo ser la opción Metronidazol $250 \mathrm{mg}$ c/ 8 horas x 7 días. ${ }^{3,5,6}$ Otras opciones son el uso de Amoxicilina de $500 \mathrm{mg}+$ Ac clavulanico de $125 \mathrm{mg}$ c/8 horas $\mathrm{x} 7 \mathrm{dí}^{7}$. A todo lo mencionado, es de vital importancia, que el paciente mejore su higiene bucal, para lo cual se le enseña una fisioterapia adecuada para el correcto uso del cepillado e hilo dental y demás aditamentos para el mejor cuidado de su boca. ${ }^{17}$

2. Tratamiento mediato, llevada al día siguiente y citas periódicas de cada 2 días después de la 2 da cita. Aquí se trata de completar el desbridamiento mecánico, realizando un raspaje y alisado en toda la dimensión del surco. Evaluar los resultados y ver la mejora del paciente. Esta etapa debe darse hasta que los signos y síntomas se resuelvan, restaurándose la salud y función gingival. ${ }^{5,17,21}$ En esta fase también se trata de concientizar al paciente en dejar o reducir hábitos como el alcohol y tabaco. ${ }^{6}$ Mención importante tendría la GN presente en paciente con el virus de la inmunodeficiencia humana (VIH), acotando que su presencia se da por lo general en el tercer estadio de la enfermedad, según clasificación de la Organización Mundial de la Salud (OMS) y que estaría relacionada a una baja en el conteo de células CD4 de 500 CD4/ $\mathrm{mm} 3$ a $200 \mathrm{CD} 4 / \mathrm{mm} 3$ en sangre. , $8,22,23$ $\mathrm{Su}$ tratamiento es básicamente como se ha mencionado, considerando la bioseguridad del caso, ya que el riesgo de contagio por exposición percutánea a sangre contaminada es de $0.3 \%{ }^{2}$. La medicación profiláctica a base de antimicrobianos debe ser evaluada.

\section{Periodontitis necrotizante (PN)}

Sinónimos: Periodontitis ulcero necrotizante, Periodontitis fusoespiroquetal.

\section{Definición:}

Proceso inflamatorio de origen infeccioso, caracterizado por necrosis de los tejidos gingivales, ligamento, hueso alveolar; generando una decapitación de papila y denudación del hueso alveolar interdental, la cual induce a secuestro óseo. ${ }^{6,7,8,10,23,24}$

Su presencia se da en jóvenes adultos de 18 a 30 ańos aproximadamente y por lo general con desnutrición o con inmunosupresión. ${ }^{25}$

\section{Etiología}

$\mathrm{Su}$ origen por lo general es una secuela de la GN, la cual, pudo haber sido tratado en su momento, pero no curado, la nueva presentación trae el agravante del daño óseo. En su origen también el factor microbiano es el de mayor relevancia, siendo los bacilos gram negativo, anaerobios estrictos, lo que van destruyendo los tejidos periodontales, con una deficiente respuesta por parte del huésped. ${ }^{24,25}$

\section{Fisiopatología}

Los microorganismos presentes en la lesión van destruyendo ligamento periodontal, gingiva y hueso en forma rápida y progresiva, generando grandes recesiones, pérdida de papila interdental, exposición de hueso y movilidad de diferente grado.

Esta patología presenta factores que predisponen a la $\mathrm{PN}$, agregado a los factores predisponentes que son; estrés severo, secuela o múltiples episodios de GN, severa desnutrición, fumadores crónicos e inmunodeprimidos como los pacientes con VIH, los cuales con conteo menor a $200 \mathrm{CD} 4 / \mathrm{mm}^{3}$, son más propensos a desarrollar esta lesión. ${ }^{24-26}$

\section{Microbiología}

Se han podido aislar de estas lesiones microorganismos como; Fusobacterium, Selenomonas, Prevotella intermedia, Porphyromonas gingivalis, Treponema sp y Candida albicans, pudiendo este último, verse incrementado en pacientes con VIH. ${ }^{24,25}$

\section{Clínica}

Dolor intenso, cráteres con denudación del hueso alveolar interdental, secuestros óseos, sangrado espontáneo, bolsas profundas, halitosis, son signos menos comunes linfoadenopatías, fiebre, malestar general. ${ }^{6}$ 


\section{Diagnóstico}

Basándose en los signos y síntomas casi patognomónicos de la enfermedad, así como descartando patologías similares, por diagnóstico diferencias, como; estomatitis aftosa recurrente, herpangina, mucositis microbiana eritema exudativo multiforme, ulceras traumáticas, lesiones herpéticas. ${ }^{6,7}$

\section{Tratamiento}

Realizado en 2 tiempos:

I tratamiento inmediato, anestesia local tópica de la zona, limpieza superficial con clorhexidina al $0.12 \%$, retirando restos de tejidos. Iniciar un desbridamiento cuidadoso del surco periodontal, instruir al paciente en su fisioterapia; manejo de cepillo e hilo dental. Indicar un colutorio a base de clorhexidina al $0.12 \% \times 10$ días, luego a una concentración de $0.05 \%$ como mantenimiento, medicación sistémica, que puede ser amoxicilina $500 \mathrm{mg} \mathrm{c} / 8$ horas $\mathrm{x} 7$ días, metronidazol $250 \mathrm{mg} \mathrm{c} / 8 \mathrm{~h} \mathrm{x} 7$ días o su combinación ${ }^{25}$. Otras alternativas, son usar clindamicina o amoxicilina más ácido clavulanico. ${ }^{4,7}$ En caso de pacientes inmunocomprometidos evaluar el riesgo de sobreinfección por cándida, para tal caso medicar antifúngicos.

II Tratamiento mediato, complementar el desbridamiento a profundidad, se realiza a las 24 horas, luego se da cita cada 48 horas para evaluar los signos y síntomas del paciente. Así como su control de placa. Esto hasta controlar lo más agudo de la enfermedad. Recomendamos su derivación al periodoncista, como a un médico internista y nutricionista para ver su condición general y posible desnutrición respectivamente. ${ }^{25}$

Mención especial tiene lo pacientes con VIH, con presencia de PN, donde los procedimientos deben ser mínimamente invasivos, evaluando los resultados cada 24 horas. Recordando que estos pacientes con PN, presentan el $72.9 \%$ de probabilidad acumulada de muerte dentro de las 24 meses de la aparición de la $\mathrm{PN}^{25}$, así como un riesgo de desarrollar Noma.

\section{Estomatitis necrotizante (EN)}

Sinonimos: Estomatitis ulcero necrotizante, Noma, Gangrena fusoespiroquetal, Cancrum oris, Face of poverty.

\section{Definición}

Es una enfermedad gangrenosa, que puede desarrollarse de una GN o PN, afectando tejidos de la boca, cara y vías respiratorias, generando una úlcera necrótica destructiva, que puede llegar a deformar la boca como el rostro. ${ }^{11,12,27,28}$
Es poco frecuente, aunque en países del África, se observa en niños, estimándose una incidencia anual de 6.4 casos por 1000 niños. Varios estudios indican que los factores de riesgo que desencadenan esta enfermedad son de pobreza extrema, desnutrición, mala higiene bucal, sarampión y malas condiciones sanitarias entre los principales. ${ }^{11,28}$

\section{Etiología}

Su origen está en relación con una GN o PN, asociada a una marcada carga microbiana, así como los factores de riesgo anteriormente mencionados.

\section{Fisiopatología}

$\mathrm{Su}$ fisiopatología se respalda en 3 teorías: Teoría vascular, que generaría isquemia y trombosis arterial, los cual llevaría a la necrosis. Teoría viral, que sugiere que el virus herpes podría reducir la inmunidad local, por tanto facilitar el desarrollo de la flora bacteriana patógena. Recordando que el Citomegalovirus es el más frecuente en la enfermedad periodontal, que puede invertir el conteo de CD4/ CD8, infectar neutrófilos e interferir en su función, así como generar un incremento de IL-1, asociado a la resorción ósea. Teoría bacteriana, que es la que tiene mayor sustento, predominando la flora gram negativa, anaerobia estricta. ${ }^{11,12}$

\section{Microbiología}

Se han podido identificar en esta lesión: Prevotella melaninogénica, Corynebacterium pyogenes, Peptoestreptococcus stomatis, Prevotella intermedia y Fusobacterium necrophorum, siendo estas 2 últimas de relevancia en el Noma. ${ }^{11,27}$

\section{Clínica}

Se puede desencadenar de una GN o de una PN, la fase aguda se caracteriza por fiebre, apatía, sangrado gingival, lesión de la mucosa oral, edema de cara, olor fétido. ${ }^{19}$ Presencia de mancha o pápula que forma rápidamente una úlcera con compromiso óseo, generando al final una ulcera necrótica destructiva de la boca que en algunos casos abarca la cara. ${ }^{11}$

\section{Diagnóstico}

Identificación de lesión ulcera necrótica que se extiende más allá de los limites mucogingivalis. En la confirmación realizar diagnóstico diferencial, con lesiones parecidas presentes en; leishmaniasis mucocutánea, angina granulítica, lesión oral maligna, sífilis, úlcera de Burulli, entre otros. ${ }^{11,12}$

\section{Tratamiento}

Se da en 2 fases:

I Fase aguda; en la que se trata de mejorar la salud general, hidratación, nutrición, rehabilitación, administrar vitamina A. El tratamiento con antibióticos de amplio espectro, en el caso de niños puede ser Metronidazol (20 $\mathrm{mg} / \mathrm{Kg} /$ diariamente), así como indicar enjuagues diarios con clorhexidina al $0.12 \%$ ó $0.2 \% .^{11,12}$ Mejorar la higiene bucall del paciente, evitar la intervención invasiva intraoral, debido al riesgo de precipitar la evolución de la lesión. Realizar interconsulta con periodoncista, médico internista y nutricionista, para una completa evaluación del paciente. II Fase quirúrgica; realizada para corregir deformaciones, que se puede presentar tanto intraoral como extraoral, realizado por el cirujano plástico periodontal y médico cirujano plástico. ${ }^{11,12}$

\section{Discusión}

Las enfermedades periodontales necrotizantes según la AAP son dos, la GN y la $\mathrm{PN}$, aunque autores como Bermejo ${ }^{3}$, Salinas ${ }^{5}$, Perez-Salcedo ${ }^{6}$, Kinane ${ }^{10}$, manifiestan que estas patologías pueden ser una secuela para algo mayor, que derivaría en una EN. Su prevalencia en la actualidad es baja en países desarollados, $0.5 \%$, aunque en inicios del siglo XX y en plena segunda guerra mundial, se tenían reportes de $14 \%$ en militares daneses, esto mencionado por Jenkins $^{13}$. Lópe ${ }^{29}$ en su estudio con 9023 ciudadanos chilenos determino una prevalencia de $6.7 \%$ en GN. Aunque Perez-Salcedo ${ }^{6}$ informa en su revisión que esta prevalencia puede llegar a $26.9 \%$ aproximadamente en países muy pobres como los Africanos y por lo general asociado a edades tempranas y desnutrición. Según Enwonwu ${ }^{30}$ la desnutrición produciría alteración de nuestro sistema de respuesta, incrementando una variedad de interleuquinas como IL-8, IL-6, IL-1 $\beta$, participantes en el daño periodontal, así como un incremento del cortisol sérico que generaría una alteración de la función de linfocitos, esto también manifestado por Sabiston ${ }^{31}$ en su investigación.

Para Muruyama ${ }^{1}$ la GN es una lesión rápidamente destructiva de etiología compleja, caracterizada por necrosis de la cresta papilar. Pero investigadores como Kinane ${ }^{10}$, Armitage ${ }^{9}$, Bascones ${ }^{7}$, Simeone $^{2}$, la consideran una lesión con signos patognomónicos como el dolor, ulceración con necrosis papilar y sangrado, asociado a factores predispo- 
nentes, principalmente desnutrición o inmunosupresión.

Novak $^{24}$ en su revisión sobre PN, manifiesta que esta lesión es una secuela de la GN, con gran pérdida de adherencia clínica y hueso alveolar y que el factor microbiano se compone de Treponemas, Selenomonas, Fusobacterium nucleatum, Prevotella intermedia y Porphyromonas gingivalis. Informacion similar menciona Todescan ${ }^{25}$ en un reporte de caso clínico. Mención de importancia, es que la OMS la coloca, en las patologías de aparición en el tercer estadio de los pacientes infectados por VIH, lo cual indicaría un CD4 entre $\operatorname{los} 500 / \mathrm{mm}^{3}$ a $200 / \mathrm{mm}^{3}$, esto también mencionado en la investigación de Ramos ${ }^{8}$. Otros investigadores como Bodhade $^{26}$, Todescan $^{25}$ y Novak ${ }^{24}$, mencionan que su aparición sería más insidioso con CD4 por debajo de los $200 / \mathrm{mm}^{3}$.

En la actualidad hay pocos reportes sobre la EN, esta enfermedad se ha circunscrito principalmente en países africanos, donde pueden darse incidencias de 6.4 casos /1000 nińos, esto según Enwonwu ${ }^{12}$, aunque Baelum ${ }^{28}$ estima una prevalencia aproximada en países como Sierra leona, Gambia, Nigeria de $0.1 \%$ entre los nińos de 0 a 6 ańos. Siendo el factor pobreza, que llevaría a la desnutrición y descuido total de su cavidad bucal.

La GN está asociada principalmente a la desnutrición e inmunosupresión. Reportes en pacientes con VIH, como el de Narami $^{23}$, indicaría una posible presencia con una baja de CD4 a 400/ $\mathrm{mm}^{3}$, aunque Simeone $\mathrm{e}^{2}$ menciona que el $30 \%$ de los pacientes seropositivos al VIH lo presentan, situándose preferentemente en zonas antero inferiores, zonas de extracción, dientes apiñados o dientes con bandas ortodónticas. Tomando esto último como referencia al tratamiento ortodóntico, Sanganni ${ }^{32}$, reporta una serie de casos de GN en pacientes con tratamiento ortodóntico seronegativos, indicando un reconocimiento temprano y tratamiento precoz para evitar la pérdida irreversible de la papila interdental.

Investigadores como Bermejo ${ }^{3}$, Salinas $^{5}$, Bascones ${ }^{7}$, Atoun $^{21}$, Todescan ${ }^{25}$, Muruyana $^{1}$, Perez $^{17}$, Perez-Salcedo ${ }^{6}$, concuerdan que el tratamiento para las enfermedades periodontales necrotizantes deben ser de forma inmediata, teniendo dos etapas. La primera reducirá el biofilm, sin procedimientos invasivos a profundidad, para no dispersar ni profundizar más la flora mi- crobiana presente, para luego indicar antimicrobianos de uso local o según sea el caso de uso sistémico, a su vez mejorar la higiene del paciente, ensenándole una fisioterapia para su caso, controlar el dolor con analgésicos o antiinflamatorios. La segunda etapa debe realizarse a las 24 o 48 horas, terminando con el desbridamiento a profundidad del surco, dejando la zona lo más liso y libre de biofilm, evaluando el caso cada 48 horas, con una fase de mantenimiento permanente. Para casos graves como PN y $\mathrm{EN}$, se sugiere remitir al paciente a un periodoncista, hacer interconsulta con el médico internista, inmunólogo y nutricionista. Casos graves de $\mathrm{PN}$ se pueden asociar a severa inmunodeficiencia congénita, donde la deficiencia de inmunoglobulinas Ig G y Ig A pueden desencadenar esta patología, Batista ${ }^{33}$ en un reporte de caso, manifiesta que un tratamiento a base de inmunoglobulinas por vía endovenosa en dosis de $400 \mathrm{mg}$ cada 30 días, por un período de un año, logra mejorar las condiciones periodontales.

\section{Conclusiones}

Las enfermedades periodontales necrotizantes, tienen un origen complejo, donde se asocian factores microbianos y factores predisponentes como la desnutrición y inmunosupresión principalmente. Se considera como signos diagnóstico la ulceración de la papila de forma crateriforme con márgenes gingivales enrojecidos. Cubriendo a ésta lesión una seudomembrana constituido por un exudado fibrinoso de color gris amarillento el cual se retira fácilmente dejando una superficie sangrante y dolorosa. Su prevalencia en la actualidad es baja en países desarrollados, pero relativamente alta en países pobre como los africanos. En relación a su tratamiento este debe ser inmediato y no invasivo, remitiendo al paciente para casos más severos al periodoncista.

\section{Agradecimiento.}

A la Srta Yuli Ramos P, estudiante de nutrición por la colaboración en el tipeo de este artículo.

\section{Referencias bibliográficas}

1. Muruyama Y, Kurihara H, Nagai A, Dompkowski D, Van Dyke T. Acute Necrotizing ulcerative Gingivitis: Risk factors involving host defense mechanisms. Periodontol 2000. 1994;6:116-124.

2. Simeone GS, Castillo AC. Gingivitis ulcero necrotizante y su relación con el VIH/ SIDA. Acta Odontol Venez. 2007;45(3):473-8.

3. Bermejo FA, Sanchez PA. Enfermedades periodontales necrosantes. Med Oral Patol Oral Cir Bucal 2004;9:108-119.

4. Pitarch BG, Sanchez MJ, Pitarch AA. Enfermedad Periodontal Necrosante. Med Cutan Iber Lat Am. 2009;37(2):108-110.

5. Salinas MY, Millán IR. Enfermedad Periodontal Necrosante - Conducta odontológica. Acta odontológica Venezolana, 2009;47(4):1-25.

6. Perez-Salcedo L, Bascones MA. Formas agudas de periodontitis. Av Periodon Implantol. 2008;20(1):49-58.

7. Bascones MA, Escribano BM. Enfermedad Periodontal Necrosante: Una manifestación de trastornos sistémicos. Med clin (Barc). 2005;125(18):706-713.

8. Ramos PD. Estudio comparativo de las lesiones periodontales en gestantes VIH seropositivos y VIH no seropositivos. [Tesis de Grado de Bachiller]. Lima: Facultad de Odontología de la Universidad Nacional Mayor de San Marcos; 1999.

9. Armitage G. Diagnóstico y clasificación de las enfermedades periodontales. Periodontology 2000 (Ed Esp), 2005;9:9-21.

10. 10.- Kinane D, Podmore M, Ebersole J. Etiopathogenesis of periodontitis in children and adolescents. Periodontol 2000. 2001;26:54-91.

11. Baratti MD, Pittet B, Montandon D, Bolivar I, Etienne BJ, Hugonnet S, Jaquinet A y cols. Noma: An infectious disease of unknowm etiology. Lancet Infect Dis. 2003;3:419-431.

12. Enwonwu C, Fakler W, Phillips RS. Noma (Cancrum Oris). The Lancet. 2006;368:147-156.

13. Jenkis WM, Papapanou PN. Epidemiology of Periodontal Disease in Children and adolescents. Periodontol 2000. 2001;26:16-32. 
14. Maita VL, Castañeda MM, Maita CL. Enfermedades Periodontales Necrotizantes. Revista del COP región Lima 2010;15(32):27-30.

15. Horning GM, Cohen ME. Necrotizing ulcerative gingivitis, periodontitis and stomatitis: Clinical staging and predisposing factors. J Periodontol. 1995;66(11):990998.

16. Mac Carthy D, Claffey N, Acute Necrotizing Ulcerative Gingivitis is associated with attachment loss. J Clin Periodontal. 1991;18:776779 .

17. Pérez N, Bermúdez J, Guillen G, Suarez I. Gingivitis ulcero necrotizante reporte de un caso. Ciencia Odontológica 2010;7(1):66-72.

18. Ramos PD, Avila-Campos MJ, Levano TV. Treponema denticola: Patógeno en procesos periodontales y pulpares. Odontol Sanmarquina 2012;15(2):38-41.

19. Scully C, Greenman J. Breath odor. Periodontol 2000. 2008;48:66-75.

20. 20.- Corbet E. Diagnosis of acute periodontal lesions. Periodontol 2000. 2004;34:204-216.

21. Atout R, Todescan S. Managing patients with necrotizing ulcerative gingivitis. J Can Dent Assoc. 2013;79;46.
22. Gourlay A, Van Tienen C, Dave S, Vincent T, Rowland S, Glynn J, Whittle H, Schim VM. Clinical predictors cannot replace biological predictors in HIV-2 infections in a community setting in west Africa. International J infect diseas. 2012;16:337-343.

23. Narani N, Epstein J. Classifications of oral lesions in HIV infection . J Clin Periodontol. 2001;28:137-145.

24. Novak MJ. Necrotizing ulcerative periodontitis. Ann Periodontol.1999;4(1):74-78.

25. Todescan S, Atout R. Managing Patients with Necrotizing Ulcerative Periodontitis. J Can Dent Assoc. 2013;79:44.

26. Bodhade A, Ganvir S, Hazarey V. Oral manifestations of HIV infection and their correlations with CD4 count. J Oral Science. 2011;53(2):203-211.

27. Bolivar I, Whiteson K, Stadelmann B, Barrati MD, Gizard Y, Mombelli A, Pittet D, Schrenzel J, Bacterial diversity in oral samples of children in Niger with acute Noma, Acute Necrotizing Gingivitis and Healthy controls. Plos Negl Trop Dis. 2012;6(3):111.
28. Baelum V, Scheutz F, Periodontol diseases in Africa. Periodontol 2000. 2002;29:79-103.

29. Lopez R, Fernandez O, Jara G, Baelum V. Epidemiology of Necrotizing Ulcerative Gingival lesions in adolescent. J Peridont Res. 2002;37:439-444.

30. Enwonwu C, Phillips R, Savage K. Inflamatory cytokine profile and circulating cortisol levels in malnourished children with necrotizing ulcerative gingivitis. Eur Cytokine Netru. 2005;16(3):240248.

31. Sabiston Jr CB. A Review and proposal for the etiology of acute necrotizing gingivitis. J Clin Periodontol. 1986;13:727-734.

32. Sanganni I, Watt E, Cross D. Necrotizing ulcerative gingivitis and the orthodontic patient: A case series. J Orthod. 2013;40(1):77-80.

33. Batista Jr. EL, Novaes Jr AB, Calvano LM, Do Prado EA, Goudouris ES, Batista FC. Necrotizing ulcerative Periodontits associated with severe congenital immunodeficiency in a prepubescent subject: Clinical finding and response to intravenous immunoglobulin treatment. J Clin Periodontol 1999;26:499-504. 